\title{
Diagnóstico del estado nutricional de los pacientes con demencia tipo Alzheimer registrados en el Grupo de Neurociencias, Medellín, 2004
}

\author{
Angélica María Muñoz ${ }^{1}$, Gloria María Agudelo ${ }^{1}$, Francisco Javier Lopera ${ }^{2}$ \\ ${ }^{1}$ Escuela de Nutrición y Dietética, Grupo de Investigación en Alimentación y Nutrición Humana, Universidad \\ de Antioquia, Medellín, Colombia. \\ 2 Facultad de Medicina, Grupo de Neurociencias, Universidad de Antioquia, Medellín, Colombia.
}

Introducción. Los pacientes con demencia tipo Alzheimer presentan riesgo de deterioro del estado nutricional por cambios fisiológicos, socioeconómicos y psicológicos relacionados con la enfermedad, lo que se suma al proceso de envejecimiento.

Objetivo. Describir el estado nutricional de los pacientes con demencia tipo Alzheimer del Grupo de Neurociencias de la Universidad de Antioquia según el estadio de evolución.

Materiales y métodos. Estudio descriptivo transversal. Cada paciente fue evaluado para obtener información general, de salud, composición corporal, alimentaria, signos clínicos y aspectos psicosociales.

Resultados. Se evaluaron 77 pacientes con edad promedio de $65,5 \pm 12,8$ años, 48 de ellos con antecedentes familiares de la enfermedad y 39 con más de 60 meses de diagnóstico; el estadio más frecuente fue el moderado y el tipo de demencia más común, la familiar precoz; la enfermedad se diagnosticó antes de los 50 años en 26 pacientes. Hubo diferencias estadísticamente significativas en el índice de masa corporal, el área grasa braquial y el área magra braquial por estadio, y mayor compromiso en el estadio grave $(p=0,001, p=0,000$ y $p=0,000$ ); en todos los estadios se presentó compromiso de la reserva energética y muscular. El calcio y los folatos fueron los nutrientes más comprometidos en el consumo.

Conclusión. Aun en el estadio inicial, los pacientes presentaron compromiso del estado nutricional, evidenciado por déficit en la reserva energética y muscular, signos clínicos y consumo inadecuado de calcio y folatos. A medida que avanza la enfermedad, el compromiso de los indicadores nutricionales es mayor. Lo anterior evidencia la necesidad del cuidado nutricional individual de acuerdo con el estadio de la enfermedad.

Palabras clave: enfermedad de Alzheimer, estado nutricional, evolución clínica, composición corporal, consumo de alimentos, envejecimiento.

Nutritional condition in patients with Alzheimer-type dementia from the neurosciences' group, Medellín 2004.

Introduction. Patients suffering clinical dementia caused by Alzheimer's disease have a high risk of developing malnutrition caused by all the physiological, socio-economic and psychological changes related to the disease, together with the ageing process.

Objective. To describe the nutritional condition of a group of patients with Alzheimer's dementia according to the degree of disease severity. Patients were selected from the University of Antioquia Neurosciences Group.

Materials and methods. Descriptive cross sectional study. Patients were assessed and information was collected about their body composition, food consumption behaviour, clinical, and psychosocial variables.

Results. 77 patients were studied; their mean age was $65,5 \pm 12,8$ years. 48 had a family history of Alzheimer's disease; 39 were diagnosed more than 60 months before the study started; the most frequently found degree of severity was moderate, the most common type of dementia was the early family form. 26 began the disease process before they were 50 years old. Significant differences for Body Mass Index, brachial adipose area and brachial lean area 
were found ( $p=0,001, p=0,000, p=0,000$, respectively) between patients with the different degrees of disease severity. These parameters measures were lowest in patients with severe disease. The intake of calcium and folate was the lowest amongst the nutrients studied.

Conclusion. The nutritional state is impaired in patients suffering Alzheimer's dementia from the early stages of the disease. The most important nutritional and clinical abnormalities found were depletion of the lean and fat stores, low intake of calcium and folate and clinical signs of the disease. Changes in these parameters worsen as the disease progresses.

Keyword: Alzheimer's disease, nutritional status, clinical evolution, body composition, food consumption, ageing.

La enfermedad de Alzheimer ha ingresado recientemente al campo de la salud pública contemporánea y es la causa más frecuente de demencia en el adulto mayor (1). Para el caso colombiano, los ancianos aún representan una pequeña parte del total de la población del país, pero se está produciendo un cambio importante de la pirámide de la población, como consecuencia del aumento de la esperanza de vida y de la disminución de la tasa de natalidad.

La demencia tipo Alzheimer está asociada con trastornos cognitivos y de comportamiento y, frecuentemente, con trastornos nutricionales; existe evidencia de que el estado nutricional de los pacientes con demencia tipo Alzheimer queda comprometido durante la enfermedad (2-4). Un inadecuado estado nutricional puede afectar la condición general y el bienestar del paciente e influir en la evolución de la demencia, y, a su vez, ésta puede afectar el estado nutricional debido a que es común que los trastornos neurológicos graves alteren los mecanismos y las capacidades cognitivas y motoras necesarias para lograr una alimentación adecuada. Por otra parte, la malnutrición podría exacerbar la enfermedad o, aún más, jugar un papel etiológico importante (5).

En los pacientes con demencia tipo Alzheimer, el deterioro del estado nutricional se presenta por las características propias de la enfermedad, como la presencia de lesiones subcorticales

\section{Correspondencia:}

Gloria María Agudelo, Escuela de Nutrición y Dietética Universidad de Antioquia,

Carrera 75 № 65 -87 Bloque 44 Oficina 106 Medellín Colombia

Teléfono: (4)4259222; fax: 230-50-07

gmao@quimbaya.udea.edu.co

Recibido: 13/06/05; aceptado:27/01/06 hipotalámicas, las cuales pueden afectar los centros reguladores del apetito y los procesos metabólicos que se encargan de regular el peso y la composición corporal constante (6). La apraxia y la agnosia pueden alterar la ingesta diaria si estos pacientes no reciben ayuda para el consumo de alimentos; pueden también presentar períodos de hiperfagia, disfagia, anorexia, pérdida de las habilidades de masticación y deglución, además de tener alterada su capacidad para obtener el alimento, prepararlo y llevarlo a la boca (7).

Sabiendo del compromiso del estado nutricional que pueden presentar los pacientes con enfermedad de Alzheimer en las diferentes etapas, y de su efecto sobre la condición general y de salud, y considerando además la escasa información que existe en nuestro medio sobre el tema, se planteó este trabajo con el objetivo de describir el estado nutricional de los pacientes con DTA inscritos en el Grupo de Neurociencias de la Universidad de Antioquia mediante indicadores antropométricos, alimentarios, clínicos y de salud según el estadio de evolución de la enfermedad con el propósito de proponer intervenciones nutricionales oportunas que contribuyan a mejorar la calidad de vida de los pacientes y sus cuidadores.

\section{Materiales y métodos}

Este es un estudio descriptivo transversal cuya población estuvo constituida por todos los pacientes con diagnóstico de demencia tipo Alzheimerregistrados en el Grupo de Neurociencias de la Universidad de Antioquia, activos entre los meses de abril y septiembre del 2004. La población final fue de 77 pacientes con diagnóstico de demencia tipo Alzheimer según los criterios del Diagnostic and Statistical Manual of Mental Disorders DSM IV) y los criterios NINCOS- 
ADRDA (del inglés National Institute of Neurological and Communicative Disorders and Stroke - Alzheimer's Disease and Related Disorders Association). Para clasificar la gravedad y el estadio clínico de la enfermedad se utilizó la escala GDS ( Global deterioration scale) y el Functional Assessment Staging FAST ) de Reisberg $(8,9)$.

El estudio se se llevó a cabo en los consultorios de la sede del Grupo de Neurociencias y de la Escuela de Nutrición y Dietética de la Universidad de Antioquia; en los casos necesarios se realizaron visitas domiciliarias. Para la recolección de la información se utilizó la historia nutricional y dietética especialmente diseñada para los pacientes con demencia tipo Alzheimer; la encuesta fue diligenciada por nutricionistas dietistas entrenadas previamente. Cada paciente fue evaluado para obtener información relacionada con los aspectos que a continuación se enumeran.

\section{Recolección de información}

Información general y de salud: como fuente primaria, el cuidador proporcionó los datos generales y demográficos (sexo, edad, procedencia, estrato socioeconómico, actividad, estado civil, personas con quien vivía). La historia clínica, empleada como fuente secundaria, proporcionó datos relacionados con la salud (antecedentes familiares y personales de la DTA , diagnóstico clínico, tipo de demencia, estadio de la enfermedad y edad de inicio).

Composición corporal: se evaluó con técnicas antropométricas. Para la toma de los datos y el cálculo posterior de los índices nutricionales, se aplicaron técnicas previamente estandarizadas (10). El peso corporal se obtuvo en una balanza electrónica Detecto con capacidad de $150 \mathrm{~kg}$ y una sensibilidad de $50 \mathrm{~g}$; cada paciente fue pesado y medido sin zapatos y con el mínimo de ropa; en los casos en que no se pudo pesar, se tomó inicialmente el peso del cuidador, quien luego cargaba al paciente y por diferencia se obtenía el dato. Para el dato de la estatura se utilizó un estadiómetro portátil Seca fijado en la pared, con capacidad de $200 \mathrm{~cm}$ y un $\mathrm{mm}$ de sensibilidad, señalando el tope con una escuadra en ángulo recto y manteniendo al paciente con los hombros en posición relajada y los brazos colgantes; se utilizó la medición de la altura talón-rodilla para obtener el dato en aquellos pacientes que no podían ponerse de pie o mantenerse erguidos. La circunferencia braquial se registró con una cinta métrica de fibra de vidrio, de $0,5 \mathrm{~cm}$ de ancho con una sensibilidad de un $\mathrm{mm}$, marcando un punto medio entre el borde inferior del acromion y la apófisis del olécranon. El pliegue tricipital se midió en punto medio, en la cara posterior del brazo, mediante el uso de un adipómetro marca Harpender con $60 \mathrm{~mm}$ de capacidad y $0,2 \mathrm{~mm}$ de sensibilidad. El valor final de los datos antropométricos se obtuvo del promedio de tres mediciones.

Con los datos obtenidos, se calcularon el índice de masa corporal (IMC), el área grasa braquial y el área masa braquial. La clasificación del IMC se realizó de acuerdo con las recomendaciones de la Organización Mundial de la Salud (11), y el área grasa braquial y masa braquial de acuerdo con las referencias y fórmulas de Frisancho (12). Con lo anterior, el estado nutricional antropométrico de cada paciente se diagnosticó según su IMC y las reservas de masa magra y grasa.

Signos y síntomas clínicos: mediante examen físico se determinó la presencia de los signos y síntomas clínicos de los pacientes relacionados con el estado nutricional para detectar indicativos de déficit. Se evaluó el estado de la lengua, la dentadura, las encías, la boca, las conjuntivas, la piel y el aspecto de la grasa subcutánea.

Información alimentaria: la ingestión alimentaria se estimó por medio de tres recordatorios de 24 horas en días diferentes, incluyendo un día de fin de semana. La encuesta alimentaria se realizó previa estandarización del procedimiento; el entrevistador (nutricionista dietista) hizo énfasis en las cantidades y tipo de alimentos, así como en las preparaciones, registrando medidas e ingredientes utilizados; se utilizaron modelos alimentarios y medidas caseras para ayudar al entrevistado (persona que preparaba los alimentos) a cuantificar las cantidades de alimentos y bebidas consumidas. Para el cálculo del aporte de energía y nutrientes, se utilizó el programa computarizado de Evaluación de Consumo de Alimentos de la Escuela de Nutrición 
y Dietética de la Universidad de Antioquia, ECA 1(13), el cual incorpora la tabla de composición de alimentos colombianos (14) y algunos alimentos del Latinfoods (15). Se evaluó la ingesta de calorías, proteínas, calcio, hierro, vitaminas $\mathrm{B}_{6}, \mathrm{~B}_{12}$, ácido fólico y niacina. Los datos sobre estos nutrientes y los obtenidos en los tres recordatorios se promediaron, se tradujeron en el consumo diario de calorías y nutrientes y se compararon los valores actuales con los recomendados para cada uno de los nutrientes según las (dietary recommendation intake DRI). Para calcular el requerimiento de energía se aplicaron las siguientes ecuaciones (16):

Hombres: 662-9,53 x E + AF x (15,91 x P+539,6 x T); Mujeres: $354-6,91 \times E+A F \times(9,36 \times P+726 \times T)$, donde E: edad; AF: actividad física (sedentario: 1; leve: 1,11; moderado: 1,25; grave: 1,48$)$; $P$ : peso en kg; $\mathrm{T}$ : talla en $\mathrm{m}$; a partir de los 30 años disminuye $10 \mathrm{kcal}$ por cada año.

Para el análisis individual del consumo de energía y de nutrientes se utilizó una valoración cualitativa, la cual sugiere la siguiente clasificación (17): 1 . consumo probablemente inadecuado si el consumo es SEAR (estimate average requerimen) ${ }^{*}$; consumo probablemente adecuado si el consumo es RDA (recommended dietary allowances) ${ }^{* *}$; consumo posiblemente inadecuado si el consumo es $\leq R D A$ y $\geq E A R$.

Para el calcio, las nuevas recomendaciones se muestran como (adequate intake $\mathrm{Al})^{* * *}$, en virtud de que no se consideró que estuvieran sólidamente establecidos los requerimientos de este mineral durante las diferentes etapas del ciclo vital (17).

El consumo de los diferentes nutrientes en este estudio se basó exclusivamente en el aporte de

EAR, requerimiento promedio estimado: cantidad de un nutriente que se estima satisface el requerimiento de la mitad de las personas saludables de un grupo.

${ }^{*}$ RDA, recomendación dietética permitida: promedio diario de consumo suficiente para satisfacer las necesidades nutricionales del 97 al $98 \%$ de las personas saludables con diferentes características y estados fisiológicos.

${ }^{* *}$ IA, consumo adecuado: meta de ingestión de un determinado nutriente para un individuo. La Al se propone como alternativa de la RDA si no hay suficiente evidencia científica disponible para calcular la EAR. alimentos registrados en los tres recordatorios y no refleja estimaciones de otras fuentes, tales como suplementos o complementos nutricionales. La información sobre el consumo se completó con información sobre algunos aspectos relacionados con comportamientos y actitudes del paciente frente a las comidas y alimentos, como las preferencias, rechazos e intolerancias alimentarias, y formas más usuales de preparación; igualmente se preguntó por la presencia de dolor al tragar y el ahogo con sólidos y líquidos, características de estos pacientes a medida que avanza la enfermedad.

Información sobre aspectos psicosociales: mediante una entrevista realizada en todos los casos al cuidador, se indagó por aspectos psicosociales que pudieran comprometer de alguna forma el estado nutricional del paciente. Se evaluaron las siguientes variables: persona que preparaba los alimentos, disponibilidad de recursos económicos para la adquisición de alimentos, grado de autonomía para preparar y consumir alimentos y personas con quien el paciente acostumbraba comer.

\section{Análisis estadístico}

Los datos se analizaron en los programas Excel y SPSS 10.0; los datos alimentarios en el programa Evaluación del Consumo de Alimento ECA1 (13). La población de estudio se describió por edad, sexo, procedencia, estrato socioeconómico y estado nutricional, utilizando para las variables de tipo cuantitativo medidas de tendencia central y de dispersión (media, mediana, rango, desviación estándar); las variables cualitativas se describieron mediante frecuencias y porcentajes. El análisis entre grupos, el estadio de la enfermedad (leve, moderado y grave) y el estado nutricional se manejaron con la prueba de ANOVA y, como prueba posterior, el test de Scheffé en las variables paramétricas; en las no paramétricas, se utilizó la prueba de Kruskal Wallis. El nivel de significación definido fue de 0,05 y las hipótesis se plantearon a dos colas.

\section{Consideraciones éticas}

Cada paciente o acudiente aceptó voluntariamente participar en el estudio firmando el consentimiento 
informado aprobado por el Comité de Ética de la Escuela de Nutrición y Dietética de la Universidad de Antioquia.

\section{Resultados}

En cuanto a la información general, la población final fue de 77 pacientes; la edad promedio fue de $65,5 \pm 12,8$ años; 44 pacientes (57\%) tenían menos de 65 años de edad; 64 (83\%) pertenecían a los estratos socioeconómicos bajo y medio, y $69(90 \%)$ vivían con la familia u otras personas. En el cuadro 1 se resume la información general. Con respecto a los datos relacionados con salud y demencia tipo Alzheimer, en el cuadro 2 se describen los datos de salud relacionados con la enfermedad. Se destaca que en 32 pacientes (42\%), el antecedente personal de salud más común fue la enfermedad cardiovascular y el antecedente familiar de demencia tipo Alzheimer se encontró en 48 de ellos (62\%). El tipo de diagnóstico más común fue la demencia tipo Alzheimer familiar precoz en 35 pacientes (46 $\%)$. En cuanto al estadio, 26 pacientes (34\%) presentaban una DTA leve, 27 pacientes (35\%), moderada, y 24 (31\%), grave. De los 77 casos, en 26 pacientes (34\%), la edad de inicio había sido antes de los 50 años y en $39(51 \%)$ el diagnóstico de la enfermedad se había hecho hacía más de 36 meses.

Con respecto al estado nutricional según los indicadores antropométricos y el estadio de la enfermedad, del total de pacientes del estudio, sólo en uno fue imposible la evaluación del índice de masa corporal, del área grasa braquial y del área magra braquial debido a la dificultad para la toma del peso y la presencia de edema. En cuanto a la relación con el estadio de la enfermedad, 17 pacientes en estadio leve (65\%), 10 en el moderado (37\%) y 11 en el grave (48\%) presentaron un estado nutricional adecuado (IMC entre 18,5 y $\left.24,9 \mathrm{~kg} / \mathrm{m}^{2}\right)$; en el estadio moderado, 16 pacientes $(61,5 \%)$ presentaron sobrepeso (IMC> $\left.25 \mathrm{~kg} / \mathrm{m}^{2}\right)$, y en el grave, 10 pacientes (44\%) presentaron bajo peso $\left(\mathrm{IMC}<18,5 \mathrm{~kg} / \mathrm{m}^{2}\right)$. con relación al IMC se encontró diferencia estadísticamente significativa entre el estadio leve y el grave ( $p=0,001$, prueba post hoc) (cuadro 3 ). Respecto a la evaluación de la reserva grasa, presentaron compromiso en el estadio leve 11 pacientes (42\%), $13(49 \%)$ en el estadío moderado y $21(92 \%)$ en el grave. La reserva muscular mostró compromiso en $13(50 \%)$ de los pacientes en el estadio leve, en 15 pacientes (55\%) en el estadio moderado y en 22 pacientes (96\%) en el estadio grave. Se encontró una diferencia estadísticamente significativa entre los estadios tanto en el área de masa grasa $(p=0,000)$ como en el área de masa magra $(p=0,000)$, pues a mayor avance de la enfermedad, mayor el deterioro de la reserva grasa y muscular (cuadro 3 ).

Los datos del estado nutricional relacionados con los signos clínicos de los pacientes con demencia tipo Alzheimer, como se muestra en el cuadro 4, revelaron que de los 77 pacientes evaluados, 9 (12\%) tenían la dentadura completa, 9 (12\%) eran edéntulos totales, 10 (13\%), edéntulos parciales,

Cuadro 1. Información general de los pacientes con demencia tipo Alzheimer.

\begin{tabular}{llrr}
\hline Variable & Categoría & \multicolumn{2}{c}{ Frecuencia } \\
& & N & $\%$ \\
\hline Sexo & Femenino & 59 & 77 \\
& Masculino & 18 & 23 \\
Edad años & & 10 & 13 \\
& Entre 50 y 65 & 34 & 44 \\
& $>65$ & 33 & 43 \\
Procedencia & Norte & 27 & 35 \\
& Nordeste & 3 & 4 \\
& Valle de Aburrá & 16 & 21 \\
& Suroeste & 10 & 13 \\
& Occidente & 7 & 9 \\
& Oriente & 6 & 8 \\
Estrato & Otros & 8 & 10 \\
& Bajo & 21 & 27 \\
& Medio & 43 & 56 \\
Personas con & Alto & 13 & 17 \\
quien vive & Familia & 69 & 90 \\
Estado Civil & Otro & 8 & 10 \\
& Casado & 42 & 55 \\
& Soltero & 9 & 12 \\
& Viudo & 24 & 31 \\
& Otra & 2 & 3 \\
Actividad laboral & Ama de casa & 41 & 53 \\
& Educadores & 6 & 8 \\
& Profesionales & 5 & 7 \\
& Agricultor & 4 & 5 \\
& Otra & 27 \\
\hline
\end{tabular}


Cuadro 2. Datos relacionados con el estado de salud de los pacientes con demencia tipo Alzheimer.

\begin{tabular}{llcc}
\hline Variables & \multicolumn{2}{c}{ Categorías } & \multicolumn{2}{c}{ Frecuencia } \\
& & N & \% \\
\hline Antecedentes personales & Cardiovasculares & 32 & 42 \\
& Diabetes & 5 & 6 \\
& Otros & 17 & 22 \\
& Ninguna & 23 & 30 \\
Consumo de & Sí & 64 & 83 \\
medicamentos & No & 13 & 17 \\
Antecedentes de demencia Sí & & \\
tipo Alzheimer & No & 48 & 62 \\
& & 29 & 38 \\
& Esporádica precoz & 11 & 14 \\
& Esporádica tardía & 18 & 23 \\
& Familiar precoz & 35 & 46 \\
Estadio & Familiar tardía & 13 & 17 \\
& & 26 & 34 \\
& Leve & 27 & 35 \\
Edad de inicio de la & Moderado & 24 & 31 \\
enfermedad (años) & Grave & 26 & 34 \\
& Entre 50 y 65 & 24 & 31 \\
& $>65$ & 27 & 35 \\
Tiempo de diagnóstico & 6-12 meses & 1 & 1 \\
meses & $12-36$ & 16 & 21 \\
& $36-60$ & 21 & 27 \\
\hline & $>60$ & 39 & 51 \\
\hline
\end{tabular}

33 pacientes (42\%) usaban prótesis completa y $16(21 \%)$ presentaban edentulia parcial y prótesis; 36 pacientes $(47 \%)$ presentaban alteraciones en las encías, 17 de ellos en el estadio grave. Del total de pacientes estudiados, $42(55 \%)$ tuvieron conjuntivas pálidas y $18(23 \%)$, pérdida importante de grasa subcutánea.

Con relación a los datos sobre aspectos alimentarios y psicosociales de los pacientes con demencia tipo Alzheimer, la evaluación del consumo de alimentos utilizando el recordatorio de 24 horas en 3 días diferentes, se realizó en 68 pacientes de los 77 que participaron en el estudio; ello debido a dificultades por parte de los pacientes y cuidadores para suministrar la información. El porcentaje de adecuación de energía y proteínas se presenta en las figuras 1 y 2 . Respecto al consumo de energía, en 11 pacientes (46\%) en estadio leve predominó un adecuado consumo, mientras que en el estadio moderado predominó el déficit (10 pacientes, $44 \%$ ) y en el grave el exceso (8 pacientes, $38 \%$ ). En cuanto al consumo de proteínas, en los estadios leve y moderado predominó un porcentaje de adecuación mayor del $110 \%, 16$ pacientes (68\%) en el leve y 12 pacientes (52\%) en el moderado; en el estadio grave, 14 pacientes (67\%) presentaron un consumo adecuado de proteínas. El consumo promedio de calorías por día en hombres fue de $2.215 \pm 663 \mathrm{kcal}$ y en mujeres de $1.734 \pm 504 \mathrm{kcal}$. En cuanto a la distribución de los macronutrientes respecto al valor calórico total, el $59 \pm 8 \%$ provenían de los carbohidratos, el $26 \pm 6 \%$, de las grasas y el $15 \pm 3 \%$, de las proteínas. No se encontraron diferencias significativas respecto a la distribución del valor calórico total ni por sexo, ni por estadio de la enfermedad.

Para el resto de los nutrientes evaluados en todos los estadios, el calcio y los folatos fueron los más comprometidos, con valores por debajo de la

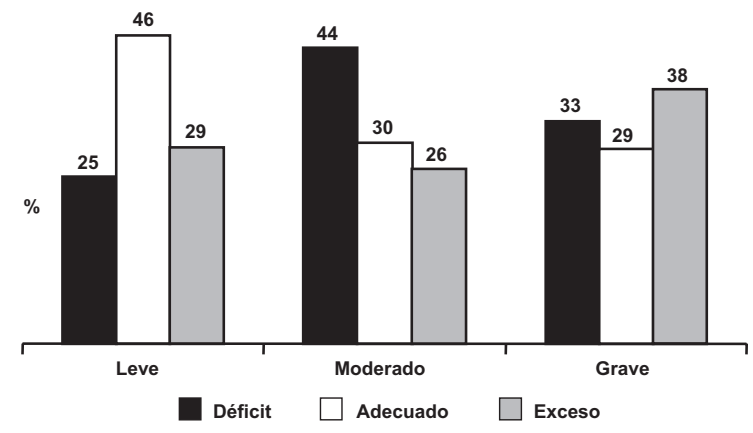

Figura 1. Porcentaje de adecuación de energía de los pacientes con demencia tipo Alzheimer según estadio.

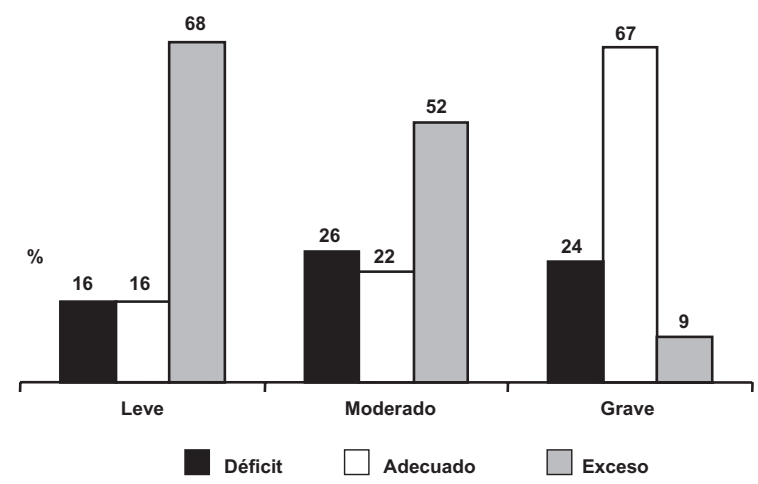

Figura 2. Porcentaje de adecuación de proteínas de los pacientes con demencia tipo Alzheimer según estadio. 
Cuadro 3. Estado nutricional según el índice de masa muscular, área grasa del brazo y área magra del brazo de los pacientes con demencia tipo Alzheimer por estadio.

\begin{tabular}{|c|c|c|c|c|c|c|c|c|c|}
\hline \multirow{3}{*}{ Estadio } & \multicolumn{9}{|c|}{ Indicador } \\
\hline & \multicolumn{3}{|c|}{ IMC (kg// $\left.{ }^{2}\right)$} & \multicolumn{3}{|c|}{ AGB $(\mathrm{mm})$} & \multicolumn{3}{|c|}{ AMB $(\mathrm{mm})$} \\
\hline & $\overline{\mathbf{X}}$ & $\mathrm{DE}$ & $\mathrm{IC}^{\mathrm{c}}$ & $\overline{\mathbf{X}}$ & DE & Med & $\overline{\mathbf{X}}$ & DE & Med \\
\hline Leve & 24,6 & 3,9 & & 2409,7 & 1295,0 & 1911,6 & 4664,8 & 1587,4 & 4177,6 \\
\hline Moderado & 24,7 & 3,1 & $-3,4 ; 2,2$ & 2323,4 & 1159,0 & 2172 & 4542,9 & 1397,4 & 4416,9 \\
\hline Grave & 19,7 & 5,6 & 1,$1 ; 7,5$ & 1395,3 & 1440,3 & 1115,2 & 3178,1 & 980,6 & 3331,1 \\
\hline$p$ & & $0,0^{a}$ & & & $0,0^{\mathrm{b}}$ & & & $0,0^{\mathrm{b}}$ & \\
\hline
\end{tabular}

IMC: índice de masa corporal. AGB: área grasa braquial. AMB: área magra braquial.

${ }^{\text {a }}$ Anova (bilateral, prueba post hoc), ${ }^{\mathrm{b}}$ Kruskall Wallis (bilateral), ${ }^{\mathrm{c}}$ intervalo de confianza del $95 \%$

Cuadro 4. Datos relacionados con los signos clínicos de los pacientes con demencia tipo Alzheimer.

\begin{tabular}{|c|c|c|c|c|c|c|c|c|c|}
\hline \multirow[t]{2}{*}{ Variables } & \multirow[t]{2}{*}{ Categorías } & \multicolumn{2}{|c|}{$\begin{array}{l}\text { Leve } \\
n=26\end{array}$} & \multicolumn{2}{|c|}{$\begin{array}{c}\text { Moderado } \\
\mathrm{n}=27\end{array}$} & \multicolumn{2}{|c|}{$\begin{array}{c}\text { Grave } \\
n=24\end{array}$} & \multicolumn{2}{|c|}{$\begin{array}{l}\text { Total } \\
\mathrm{n}=77\end{array}$} \\
\hline & & $\mathbf{N}$ & $\%$ & $\mathbf{N}$ & $\%$ & $\mathbf{N}$ & $\%$ & $\mathbf{N}$ & $\%$ \\
\hline Dentadura & $\begin{array}{l}\text { Completa } \\
\text { Edéntula total } \\
\text { Edéntula parcial } \\
\text { Prótesis completa } \\
\text { Edéntula parcial y prótesis }\end{array}$ & $\begin{array}{r}5 \\
- \\
1 \\
17 \\
3\end{array}$ & $\begin{array}{r}19 \\
- \\
4 \\
65 \\
12\end{array}$ & $\begin{array}{r}2 \\
3 \\
2 \\
14 \\
6\end{array}$ & $\begin{array}{r}7 \\
11 \\
7 \\
52 \\
22\end{array}$ & $\begin{array}{l}2 \\
6 \\
7 \\
2 \\
7\end{array}$ & $\begin{array}{r}8 \\
25 \\
29 \\
8 \\
29\end{array}$ & $\begin{array}{r}9 \\
9 \\
10 \\
33 \\
16\end{array}$ & $\begin{array}{l}12 \\
12 \\
13 \\
42 \\
21\end{array}$ \\
\hline Encías & $\begin{array}{l}\text { Sanas } \\
\text { Inflamadas } \\
\text { Sangrantes }\end{array}$ & $\begin{array}{r}20 \\
5 \\
1\end{array}$ & $\begin{array}{r}77 \\
19 \\
4\end{array}$ & $\begin{array}{r}14 \\
10 \\
3\end{array}$ & $\begin{array}{l}52 \\
37 \\
11\end{array}$ & $\begin{array}{r}7 \\
15 \\
2\end{array}$ & $\begin{array}{r}30 \\
63 \\
8\end{array}$ & $\begin{array}{r}41 \\
30 \\
6\end{array}$ & $\begin{array}{r}53 \\
39 \\
8\end{array}$ \\
\hline Conjuntivas & $\begin{array}{l}\text { Brillantes } \\
\text { Pálidas }\end{array}$ & $\begin{array}{l}15 \\
11\end{array}$ & $\begin{array}{l}58 \\
42\end{array}$ & $\begin{array}{l}13 \\
14\end{array}$ & $\begin{array}{l}48 \\
52\end{array}$ & $\begin{array}{r}7 \\
17\end{array}$ & $\begin{array}{l}29 \\
71\end{array}$ & $\begin{array}{l}35 \\
42\end{array}$ & $\begin{array}{l}45 \\
55\end{array}$ \\
\hline Alteraciones en la piel & $\begin{array}{l}\text { Sana } \\
\text { Pérdida de grasa } \\
\text { subcutánea }\end{array}$ & $\begin{array}{r}25 \\
1\end{array}$ & $\begin{array}{r}96 \\
4\end{array}$ & $\begin{array}{r}24 \\
3\end{array}$ & $\begin{array}{l}89 \\
11\end{array}$ & $\begin{array}{l}10 \\
14\end{array}$ & $\begin{array}{l}42 \\
58\end{array}$ & $\begin{array}{l}59 \\
18\end{array}$ & $\begin{array}{l}77 \\
23\end{array}$ \\
\hline
\end{tabular}

ingestión adecuada para el calcio y por debajo de los requerimientos promedio estimados para el folato; se encontraron 36 pacientes (50\%) con un consumo de vitamina $\mathrm{B}_{6}$ por debajo de los valores de las EAR. Para el hierro y la vitamina $\mathrm{B}_{12}$, el grupo estudiado alcanzó o excedió los valores de referencia de las recomendaciones dietéticas permitidas. No se encontraron diferencias estadísticamente significativas en el consumo de calorías y nutrientes de los pacientes por estadio de evolución de la enfermedad.

La disponibilidad de dinero para la compra de alimentos fue adecuada en 51 pacientes (66\%), mientras que 71 pacientes (92\%) dependían de sus familias y de otras personas para la preparación de los alimentos. En el estadio leve, todos los pacientes eran autónomos para comer, a diferencia de los pacientes en estadio grave, de los cuales, 21 (88\%) necesitaban ayuda. En todos los estadios, la forma de preparación más usual fue la de alimentos cocidos; la presencia de disfagia aumentó con el avance de la demencia. Del total de sujetos del estudio, 57 pacientes (74\%) manifestaron tener buen apetito, 62 pacientes $(81 \%)$ reportaron cambios importantes en la alimentación por la demencia, principalmente modificación de la textura para facilitar el proceso de la deglución; 22 pacientes (92\%) en estadio grave requirieron de una alimentación especial, básicamente representada en preparaciones blandas. En el cuadro 5 se resumen los aspectos anteriormente descritos. 
Cuadro 5. Distribución porcentual de características relacionadas con aspectos alimentarios de los pacientes con demencia tipo Alzheimersegún el estadio de la enfermedad.

\begin{tabular}{|c|c|c|c|c|c|c|c|c|c|}
\hline \multirow[t]{2}{*}{ Variable } & \multirow[t]{2}{*}{ Categorías } & \multicolumn{2}{|c|}{ Leve } & \multicolumn{2}{|c|}{ Moderado } & \multicolumn{2}{|c|}{ Grave } & \multicolumn{2}{|c|}{ Total } \\
\hline & & $\mathbf{N}$ & $\%$ & $\mathbf{N}$ & $\%$ & $\mathbf{N}$ & $\%$ & $\mathbf{N}$ & $\%$ \\
\hline \multirow[t]{2}{*}{ Disponibilidad económica } & Adecuada & 18 & 70 & 15 & 56 & 18 & 75 & 51 & 66 \\
\hline & Inadecuada & 8 & 30 & 12 & 44 & 6 & 25 & 26 & 34 \\
\hline Persona que compra los alimentos & $\begin{array}{l}\text { Familiares } \\
\text { Otros }\end{array}$ & $\begin{array}{r}25 \\
1\end{array}$ & $\begin{array}{r}96 \\
4\end{array}$ & $\begin{array}{r}26 \\
1\end{array}$ & $\begin{array}{r}96 \\
4\end{array}$ & $\begin{array}{r}20 \\
4\end{array}$ & $\begin{array}{l}83 \\
17\end{array}$ & $\begin{array}{l}71 \\
6\end{array}$ & $\begin{array}{c}92 \\
8\end{array}$ \\
\hline Persona que prepara los alimentos & $\begin{array}{l}\text { Familiares } \\
\text { Otros } \\
\text { El paciente }\end{array}$ & $\begin{array}{r}17 \\
2 \\
7\end{array}$ & $\begin{array}{r}65 \\
8 \\
27\end{array}$ & $\begin{array}{r}16 \\
1 \\
10\end{array}$ & $\begin{array}{r}59 \\
4 \\
37\end{array}$ & $\begin{array}{r}13 \\
- \\
11\end{array}$ & $\begin{array}{r}54 \\
- \\
46\end{array}$ & $\begin{array}{c}46 \\
3 \\
28\end{array}$ & $\begin{array}{c}60 \\
4 \\
36\end{array}$ \\
\hline Dificultad para preparar alimentos & $\begin{array}{l}\text { Sí } \\
\text { No }\end{array}$ & $\begin{array}{r}20 \\
6\end{array}$ & $\begin{array}{l}77 \\
23\end{array}$ & 27 & 100 & 24 & 100 & $\begin{array}{c}71 \\
6\end{array}$ & $\begin{array}{c}92 \\
8\end{array}$ \\
\hline Autonomía para consumir alimentos & $\begin{array}{l}\text { Necesita ayuda } \\
\text { Solo con dificultad } \\
\text { Solo sin dificultad }\end{array}$ & $\begin{array}{r}- \\
3 \\
23\end{array}$ & $\begin{array}{r}- \\
12 \\
88\end{array}$ & $\begin{array}{r}3 \\
3 \\
21\end{array}$ & $\begin{array}{l}11 \\
11 \\
78\end{array}$ & $\begin{array}{r}21 \\
2 \\
1\end{array}$ & $\begin{array}{r}88 \\
8 \\
4\end{array}$ & $\begin{array}{c}24 \\
8 \\
45\end{array}$ & $\begin{array}{l}31 \\
10 \\
59\end{array}$ \\
\hline Come & $\begin{array}{l}\text { Solo } \\
\text { Acompañado }\end{array}$ & $\begin{array}{r}7 \\
19\end{array}$ & $\begin{array}{l}27 \\
73\end{array}$ & $\begin{array}{r}4 \\
23\end{array}$ & $\begin{array}{l}15 \\
85\end{array}$ & $\begin{array}{r}2 \\
22\end{array}$ & $\begin{array}{r}8 \\
92\end{array}$ & $\begin{array}{l}13 \\
64\end{array}$ & $\begin{array}{l}17 \\
83\end{array}$ \\
\hline $\begin{array}{l}\text { Forma de preparación de alimentos } \\
\text { más utilizada }\end{array}$ & $\begin{array}{l}\text { Fritos } \\
\text { Cocinados } \\
\text { Fritos y cocidos }\end{array}$ & $\begin{array}{r}2 \\
15 \\
9\end{array}$ & $\begin{array}{r}8 \\
58 \\
35\end{array}$ & $\begin{array}{r}1 \\
16 \\
10\end{array}$ & $\begin{array}{r}4 \\
59 \\
37\end{array}$ & $\begin{array}{r}1 \\
21 \\
2\end{array}$ & $\begin{array}{r}4 \\
88 \\
8\end{array}$ & $\begin{array}{c}4 \\
52 \\
21\end{array}$ & $\begin{array}{c}5 \\
68 \\
27\end{array}$ \\
\hline Dolor para tragar & $\begin{array}{l}\text { Sí } \\
\text { No } \\
\text { Ocasionalmente }\end{array}$ & $\begin{array}{r}- \\
25 \\
1\end{array}$ & $\begin{array}{r}- \\
96 \\
4\end{array}$ & $\begin{array}{r}- \\
26 \\
2\end{array}$ & $\begin{array}{r}- \\
96 \\
7\end{array}$ & $\begin{array}{r}7 \\
15 \\
8\end{array}$ & $\begin{array}{l}30 \\
62 \\
33\end{array}$ & $\begin{array}{c}7 \\
66 \\
11\end{array}$ & $\begin{array}{c}9 \\
86 \\
14\end{array}$ \\
\hline Ahogo con líquidos & $\begin{array}{l}\text { Sí } \\
\text { No } \\
\text { Ocasionalmente }\end{array}$ & $\begin{array}{r}- \\
25 \\
1\end{array}$ & $\begin{array}{r}- \\
96 \\
4\end{array}$ & $\begin{array}{r}- \\
25 \\
2\end{array}$ & $\begin{array}{r}- \\
93 \\
7\end{array}$ & $\begin{array}{l}8 \\
8 \\
8\end{array}$ & $\begin{array}{l}33 \\
33 \\
33\end{array}$ & $\begin{array}{c}8 \\
58 \\
11\end{array}$ & $\begin{array}{l}11 \\
75 \\
14\end{array}$ \\
\hline Ahogo con sólidos & $\begin{array}{l}\mathrm{Si} \\
\text { No } \\
\text { Ocasionalmente }\end{array}$ & $\begin{array}{r}- \\
25 \\
1\end{array}$ & $\begin{array}{r}- \\
96 \\
4\end{array}$ & $\begin{array}{r}- \\
25 \\
2\end{array}$ & $\begin{array}{r}- \\
93 \\
7\end{array}$ & $\begin{array}{r}8 \\
10 \\
6\end{array}$ & $\begin{array}{l}33 \\
42 \\
25\end{array}$ & $\begin{array}{c}8 \\
60 \\
9\end{array}$ & $\begin{array}{l}10 \\
78 \\
12\end{array}$ \\
\hline
\end{tabular}

De los 77 pacientes, 17 (22\%) indicaron intolerancia alimentaria, en su orden, a la leche, las vísceras, el aguacate y los fríjoles. Entre los alimentos que más rechazaban los pacientes en los estadios leve y moderado se encontraban las frutas y las verduras. Del total de los pacientes que indicaron preferencia por algún alimento se destaca el gusto por el sabor dulce en 37 pacientes (48\%).

\section{Discusión}

El estado nutricional de los pacientes con se ve comprometido tanto por los cambios que se producen a lo largo de la enfermedad como por aquellos inherentes al proceso de envejecimiento. El deterioro cognitivo genera en los pacientes síntomas que afectan directa o indirectamente el estado nutricional (18), situación corroborada en este estudio.

Los pacientes estudiados pertenecían en su mayoría a los estratos socioeconómicos medio y bajo, situación importante por las dificultades económicas que puede representar para ellos y sus familias, pues el tratamiento de esta patología demanda cuidados especiales durante todo el proceso $(19,20)$. El mayor porcentaje de los pacientes residía en la zona norte de Antioquia, datos que concuerdan con hallazgos de otros estudios que reportan una prevalencia alta de demencia tipo Alzheimer en esta zona del departamento (21). Los pacientes en su mayoría 
viven acompañados, lo que indica, por una parte su gran dependencia para realizar actividades, entre ellas, la alimentación, y por otra, que el grupo de cuidadores constituye una pieza fundamental en todo el tratamiento de los pacientes, especialmente en el cuidado nutricional (22).

El antecedente personal de salud más común fue la cardiopatía, lo cual puede comprometer aún más la condición nutricional y de salud general de los pacientes. Algunos autores han sugerido que los componentes individuales de la cardiopatía, como el colesterol alto o la hipertensión, podrían acelerar los procesos de la enfermedad de Alzheimer debido a la restricción de fluido sanguíneo al cerebro, con la consecuente muerte neuronal a lo largo del tiempo. Además, señalan que la enfermedad de Alzheimer también afecta la pared de los vasos sanguíneos, lo cual incrementa el riesgo de ictus (Alzheimer's Association. The $9^{\text {th }}$ International Conference on Alzheimer's disease and Related Disorders: Obesity, Diet, Inactivity Linked to Dementia Risk, Pennsylvania 2004) $(23,24)$.

En estudios previos, el Grupo de Neurociencias de la Universidad de Antioquia ha identificado y estudiado el grupo de población más grande del mundo con una forma de Alzheimer familiar precoz hereditaria, conformado por 24 genealogías con más de 5.000 herederos distribuidos por todo el departamento de Antioquia (21). Coincidente con estos resultados, en este trabajo se encontró que de los 77 pacientes, en 26 la enfermedad se inició antes de los 50 años; estos datos son contrarios a las investigaciones que señalan que la mayoría de los casos de demencia tipo Alzheimer se encuentra en el decenio de los 60 años o después (25). El hecho de que el $80 \%$ de los pacientes lleva más de 36 meses con la enfermedad, refleja la necesidad de una atención nutricional temprana, pues si los pacientes con Alzheimer reciben un cuidado oportuno y adecuado, se puede prevenir la pérdida de peso y otras complicaciones (26).

En los estadios leve y grave, un mayor porcentaje de pacientes presentaba un estado nutricional adecuado según el IMC, mientras que en el estadio moderado se registró sobrepeso. Aunque este estudio fue descriptivo, los hallazgos concuerdan con trabajos que han mostrado ganancias mayores de $5 \%$ con respecto al peso inicial en pacientes con enfermedad de Alzheimer en los estadios leve y moderado (2). Coincidiendo con estudios recientes, se encontró una diferencia estadísticamente significativa entre el índice de masa corporal y el estadio, pues a mayor avance de la enfermedad, mayor es el deterioro $(27,28)$. Sato y cols reportaron que las fracturas de cadera fueron más comunes en aquellos pacientes con demencia tipo Alzheimer que tenían un índice de masa corporal bajo (29). La pérdida de peso corporal es un indicador de desnutrición y factor de riesgo para otras enfermedades como infecciones, escaras y caídas, (30). Con relación a la evaluación de la reserva grasa y muscular por antropometría braquial, se encontró que mientras más avanzada la enfermedad, los pacientes presentaron mayor deterioro. En el estadio grave, la mayoría presentaron compromiso de estas reservas, resultado similar al de otros trabajos que han señalado que los pacientes con enfermedad de Alzheimer tienen menor masa libre de grasa y masa grasa que sus controles (31). Se han postulado diversas hipótesis para explicar la pérdida de peso en los pacientes con enfermedad de Alzheimer: cambios en el consumo de alimentos asociado con la disminución de las capacidades funcionales, cambios en el requerimiento energético por agitación, estrés y deambulación, atrofia de la corteza temporal mesial y, recientemente, se han involucrado trastornos neurológicos relacionados con el neuropéptido $Y$, las citocinas y la colecistocinina, entre otros. Aunque otras investigaciones no han encontrado diferencia en el gasto energético de estos pacientes comparados con grupos control, se requieren más estudios para comprobar o rechazar dichas hipótesis $(32,33)$.

Respecto a los signos clínicos, se encontró que a medida que avanzó la enfermedad se presentó mayor compromiso, específicamente relacionado con la cavidad bucal (dentadura y encías). Algunas investigaciones han identificado patologías como ulceraciones orales en la mucosa alveolar y cara ventral lingual, frecuentemente autoinducidas por trauma con tenedores, cucharas y objetos 
extraños; se ha observado también que la presencia de prótesis dentales removibles predispone a trastornos de las mucosas y que la mala higiene oral en quienes tienen dientes naturales hace que los pacientes con Alzheimer presenten un porcentaje de placa por encima del $75 \%$ comparados con un grupo control sin demencia (11\%), lo cual se puede manifestar finalmente en gingivitis $(34,35)$. Los síntomas descritos pueden afectar el consumo de alimentos del paciente en cantidad y calidad.

La evaluación del consumo de calorías y nutrientes mostró resultados que corroboran otros hallazgos, los cuales sugieren que la pérdida de peso, común en estos pacientes, no se relaciona directamente con la ingestión de energía y nutrientes $(36,37)$. Respecto al consumo de calorías, el mayor compromiso se presentó en los pacientes en el estadio moderado, aunque no necesariamente en éste predominó el déficit de peso según el indicador IMC, ya que incluso fue más frecuente el sobrepeso. En un estudio realizado en pacientes con demencia tipo Alzheimer, Kwan y col. encontraron que el consumo de calorías y proteínas era mayor que en el grupo control (38); otro trabajo reportó que, especialmente quienes presentaron pérdida de peso consumieron más calorías por $\mathrm{kg}$ de peso por día (39). El consumo de proteínas en todos los estadios mostró un porcentaje de adecuación apropiado, incluso mayor de $110 \%$. A pesar de esto y de que el aporte al valor calórico total de este nutriente estuvo dentro del rango esperado, los pacientes mostraron una tendencia al déficit en la reserva de masa magra y masa grasa, especialmente en los paciente en estadio más avanzado, observándose en ellos un compromiso importante en el consumo calórico. Lo anterior podría indicar, entre otros aspectos, que a pesar del adecuado aporte de proteínas, éstas no cuentan con el suficiente respaldo calórico y se desvían de su función principal para servir como fuente energética. Diversas investigaciones han sugerido que otros factores diferentes al consumo son los responsables de la pérdida de masa muscular en estos pacientes; se propone un aumento en el gasto energético total por estados hipermetabólicos, hiperactividad y disminución de la actividad física, entre otros (31).

De los micronutrientes analizados, el calcio y el folato fueron los más comprometidos, junto con la vitamina $\mathrm{B}_{6}$. Es conocida la tendencia de los pacientes con enfermedad de Alzheimer a las caídas, las fracturas y la prevalencia de osteoporosis $(40,41)$. Por lo anterior, y con los resultados del estudio, queda clara la importancia de vigilar en estos pacientes el consumo de calcio, tanto para tratar las deficiencias como para prevenir complicaciones. De otro lado, estudios anteriores han sugerido que las altas ingestiones de folato y de vitaminas $B_{6}$ y $B_{12}$ se relacionan con un menor riesgo con demencia tipo Alzheimer, aunque los reportes son inconsistentes (42-44). La deficiencia de folato, y de vitaminas $B_{12}$ y $B_{6}$ puede resultar en altas concentraciones de homocisteína en el plasma, lo cual se relaciona con un mayor riesgo de cardiopatía coronaria y arteriopatía periférica $(45,46)$; la hiperhomocisteinemia se ha reportado hasta en $7 \%$ de quienes padecen la enfermedad de Alzheimer (47).

La presencia de disfagia tanto con líquidos como con sólidos aumentó con el avance de la demencia. En los pacientes con demencia tipo Alzheimer, dichas alteraciones tienen consecuencias importantes como deshidratación, asfixia, congestión, desnutrición, infecciones recurrentes en el tracto respiratorio, neumonía por aspiración y hasta la muerte; el riesgo de neumonía también se ha relacionado con el estado nutricional y la respuesta inmune de los pacientes. Aunque escasa, hay evidencia que muestra que el manejo apropiado de la disfagia puede prevenir la neumonía (48).

El estudio encontró que, a medida que avanza la enfermedad, los pacientes reciben sin ninguna dificultad lo ofrecido, factor positivo para alcanzar el requerimiento de calorías y nutrientes y evitar déficit nutricionales. El grupo de alimentos que más rechazaron los pacientes fueron las frutas y las verduras, lo cual hace necesario establecer alternativas para mejorar su consumo y contribuir a un adecuado aporte de vitaminas, minerales y fibra. En otros trabajos se encontraron alteraciones en el apetito y cambios importantes 
en la alimentación que se hacen más drásticos a medida que avanza la enfermedad (40).

\section{Conclusiones}

Los resultados del estudio muestran claramente la importancia de evaluar de manera integral el estado nutricional de los pacientes con demencia tipo Alzheimer con base en todos los indicadores y en todos los estadios. El aumento de la prevalencia de la demencia tipo Alzheimer debe ser una alerta para que los equipos de salud se preparen para su manejo multidisciplinario y, de manera especial, el profesional en nutrición y dietética, quien tiene la responsabilidad de brindar un óptimo soporte nutricional durante todas las etapas de la enfermedad y contribuir de esta manera a mantener un estado nutricional adecuado y a prevenir las complicaciones para contribuir a mejorar la calidad de vida tanto de los pacientes como de sus cuidadores. De hecho hay evidencia de que los pacientes pueden mantener el peso si tienen una dieta con un adecuado aporte calórico y de nutrientes (49). El estudio descriptivo realizado con los pacientes con demencia tipo Alzheimer del Grupo de Neurociencias de la Universidad de Antioquia puede tener limitaciones por no haber incluido la evaluación bioquímica de los nutrientes de interés y una evaluación de la composición corporal con métodos más exactos como la bioempedancia y la (densitometría axial computarizada DEXIA). Sin embargo, sus resultados constituyen un punto de partida y podrán contribuir a orientar futuros trabajos que incluyan estas variables y tengan como objetivo dar respuesta a las preguntas relacionadas con el abordaje nutricional de los pacientes con demencia tipo Alzheimer .

\section{Agradecimientos}

Muy especialmente a los pacientes, familiares y cuidadores, sin quienes no hubiese sido posible realizar este estudio.

\section{Conflicto de intereses}

Los autores manifestamos que no existe ningún conflicto de intereses, ni real ni potencial, en torno a los resultados presentados.

\section{Financiación}

Universidad de Antioquia

\section{Referencias}

1. Jacquier M. Prevalencia de las demencias con énfasis en la enfermedad de Alzheimer en la población comunitaria de Santa Fe de Bogotá. Bogota: Secretaría Distrital de Salud; 2000.

2. White H, Pieper C, Schmader K, Fillenbaum G. A longitudinal analysis of weight change in Alzheimer's disease. J Am Geriatr Soc 1995;43:SA5.

3. Guyonnet S, Nourhashemi F, Andrieu S, Ousset PJ, Gray LK, Fitten LJ et al. A prospective study of changes in nutritional status in Alzheimer's patients. Arch Gerontol Geriatr 1998;26(suppl. 1):255-62.

4. Berlinger WG, Potter JF. Low body mass index in demented outpatients. J Am Geriatr Soc 1991;39: 973-8.

5. Solís EA, Islas J, Siller JJ, Franco MO, Silva JGI, Hernández T. Aspectos nutricios de la enfermedad de Alzheimer [revista en línea]. Neurol Neurocir Psiquiatr 2002;35:150-5. Disponible en: www.imbiomed. com.mx/ Neurosiqui/Npv35n3/espanol//nicio.html

6. Sparks D, DeKosky ST, Markesbery W. Alzheimer's disease: aminergic-cholinergic alterations in hypothalamus. Arch Neurol 1988;45:994-9.

7. Harris NG. Nutrición en la vejez. En: Mahan LK, EscoteStump S, editores. Nutrición y dietoterapia de Krause. Décima ed. Ciudad de México: Mc Graw-Hill Interamericana editores S.A de C.A; 2001. p.319-20.

8. Reisberg B. Functional assessment staging (FAST). Psychopharmacol Bull 1988;24:653-9.

9. Reisberg B, Ferris SH, de Leon MJ, Crook T. The Global Deterioration Scale for assessment of primary degenerative dementia. Am J Psychiatry 1982;139: 1136-9.

10. Lohman TG, Roche AF, Martorell R. Anthropometric standardization referent manual. Champaign: Human Kinetics Books; 1988. p.171.

11. Organización Mundial de la Salud. El estado físico: uso e interpretación de la antropometría, Serie de Informes Técnicos, 854. Ginebra: Organización Mundial de la Salud; 1995. p.357-459.

12. Frisancho AR. New norms of upper limb fat and muscle areas for assessment of nutritional status. Am J Clin Nutr 1981;34:2540-5.

13. Manjarrés LM, Correa JM. Software de análisis de consumo de alimentos ECA 1. Medellín: 2004.

14. Instituto Colombiano de Bienestar Familiar. Tabla de Composición de Alimentos Colombianos. 4ㄹ ed. Bogotá: Ministerio de Salud Pública; 1978.

15. FAO/LATINFOOD. Tabla de composición de alimentos de América Latina 2002. Ingress Communications. Disponible en: http://www.rlc.fao.org/bases/alimento. 
16. Institute of Medicine of the National Academies. Energy, carbohydrate, fiber, fat, fatty acids, cholesterol, protein, and amino acids. Summary and chapters 1 through. Washington, D.C.: The National Academies Press; 2002.

17. Murphy SP, Barr SI, Poos MI. Using the new dietary reference intakes to assess diets: a map to the maze. Nutr Rev 2002;60:267-75.

18. White HK, McConnell ES, Bales CW, Kuchibhatla M. A 6-month observational study of the relationship between weight loss and behavioural symptoms in institutionalized Alzheimer's disease subjects. J Am Med Dir Assoc 2004;5:89-97.

19. Arno PS, Levine C, Memmott, MM. The economic value of informal caregiving. Health Aff 1999;18: 182-8.

20. Ernst RL, Hay JW. The US economic and social cost of Alzheimer's disease revisited. Am J Public Health 1994;84:1261-4.

21. Lopera F. Alzheimer y otros trastornos neurodegenerativos en Antioquia. Innovación y Ciencia 1999; 8:80.

22. Diwan S, Hougham GW, Sachs GA. Strain experienced by caregivers of dementia patients receiving palliative care: findings from the Palliative Excellence in Alzheimer Care Efforts (PEACE) Program. J Palliat Med 2004;7:797-807.

23. Langa KM, Foster NL, Larson EB. Mixed dementia: emerging concepts and therapeutic implications. JAMA 2004;292:2901-8.

24. Luchsinger J, Mayeux R. Cardiovascular risk factors and Alzheimer's disease. Curr Atheroscler Rep 2004;6:261-6.

25. National Institute on Aging. La enfermedad de Alzheimer en el 2000. Rev Panam Salud Pública 2001;10:268-76. Disponible en: URL http:// www.scielosp.org/scielo.php? script=sci arttext\& pid=S1020-49892001001000012\&Ing=en\&nrm=iso

26. Riviere S, Gillete-Guyunnet S, Voisin T, Reynish E, Andrieu $S$, Lauque $S$ et al. A nutritional education program could prevent weight loss and show cognitive decline in Alzheimer's disease. J Nutr Health Aging 2001;5:295-9.

27. White $\mathbf{H}$, Pieper $\mathbf{C}$, Schmader K. The association of weight change in Alzheimer's disease with severity of disease and mortality: a longitudinal analysis. J Am Griatr Soc 1998;46:1223-7.

28. Prasher $\mathbf{P}$, Metseasgharun $\mathbf{T}$, Haque $\mathbf{S}$. Weight loss in adults with Down syndrome and with dementia in Alzheimer's disease. Res Dev Disabil 2004;25:1-7.

29. Sato S, Kanoko T, Satoh K, Iwamoto J. Risk factors for hip fracture among elderly patients with Alzheimer's disease. J Neurol Sci 2004;223:107-12.
30. Pinchcofsky-Devin GD, Kaminski MV Jr. Correlation of pressure sores and nutritional status. J Am Geriatr Soc 1989;34:435-40.

31. PoehIman ET, Dvorak R. Energy expenditure, energy intake, and weight loss in Alzheimer disease. Am J Clin Nutr 2000;71:650S-5S.

32. Riviere S, Guyonnet SG, Nourhashemi F, Vellas B. Nutrition and Alzheimer's disease. Nutr Rev 1999; 57:363-7.

33. Grundman M, Corey-Bloom J, Jernigan $T$, Archibald S, Thal LJ. Low body weight in Alzheimer's disease is associated with mesial temporal cortex atrophy. Neurology 1996;46:1585-91.

34. Ship JA. Oral health of patients with Alzheimer's disease. J Am Dent Assoc 1992;123:53-8.

35. Ship JA, Puckett SA. Longitudinal study on oral health in subjects with Alzheimer's disease. J Am Geriatr Soc 1994;42:57-63.

36. PoehIman ET. Regulation of energy expenditure in aging humans. J Am Geriatr Soc 1993;41:552-9.

37. Razay G, Gordon KW. Hyperinsulinaemia and Alzheimer's disease. Age Ageing 1994;23:396-9.

38. Kwan M, Kwok T, Lam L, Woo J, Chiu H. A pilot study of associated factors of weight changes in community-dwelling patients with Alzheimer's disease. Nutr Res 2005;25:111-8 .

39. Wang PN, Yang CL, Lin KN, Chen W T, Chwang LC, Liu HC. Weight loss, nutritional status and physical activity in patients with Alzheimer's disease. J Neurol 2004;251:314-20.

40. Johansson C, Skoog I. A population-based study on the association between dementia and hip fractures in 85-year olds. Aging 1996;8:189-96.

41. Melton LJ 3rd, Beard CM, Kokmen E, Atkinson EJ, O'Fallon WM. Fracture risk in patients with Alzheimer's disease. J Am Geriatr Soc 1994:42:614-9.

42. Basun H, Fratiglioni L, Winblad B. Cobalamin levels are not reduced in Alzheimer's disease: results from a population-based study. J Am Geriatr Soc 1994;2:1326.

43. Joosten E, Lesaffre E, Riezler R, Ghekiere V, Dereymaeker L, Pelemans W, et al. Is metabolic evidence for vitamin B-12 and folate deficiency more frequent in elderly patients with Alzheimer's disease? J Gerontol A Biol Sci Med Sci 1997;52:M76-9.

44. Lindeman RD, Romero LJ, Koehler KM, Liang H, LaRue A, Baumgartner RN et al. Serum vitamin B12, $\mathrm{C}$ and folate concentrations in the New Mexico elder health survey: correlations with cognitive and affective functions. J Am Coll Nutr 2000;19:68-76.

45. Welch G, Loscalzo J. Homocysteine and atherothrombosis. N Engl J Med 1998;338:1042-50. 
46. Seshadri S, Wolf PA. Homocysteine and the brain: vascular risk factor or neurotoxin? Lancet Neurol 2003;2:11.

47. Seshadri S, Beiser A, Sehub J, Jacques PF, Rosenberg IH, D'Agostino RB et al. Plasma homocysteine as a risk factor for dementia and Alzheimer's disease. N Eng J Med 2002;346:476-83.
48. Kalia M. Dysphagia and aspiration pneumonia in patients with Alzheimer's disease. Metabolism 2003;10(Supp 2):36-8.

49. Spindler A, Renvall M, Nichols J, Ramsdell JW. Nutritional status of patients with Alzheimer's disease: a 1-year study. J Am Diet Assoc 1996;96:1013-8.

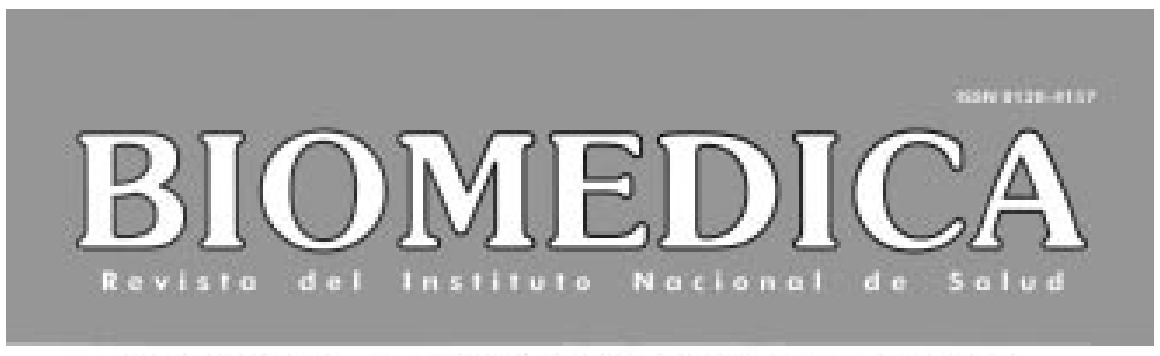

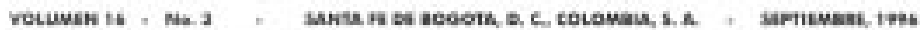

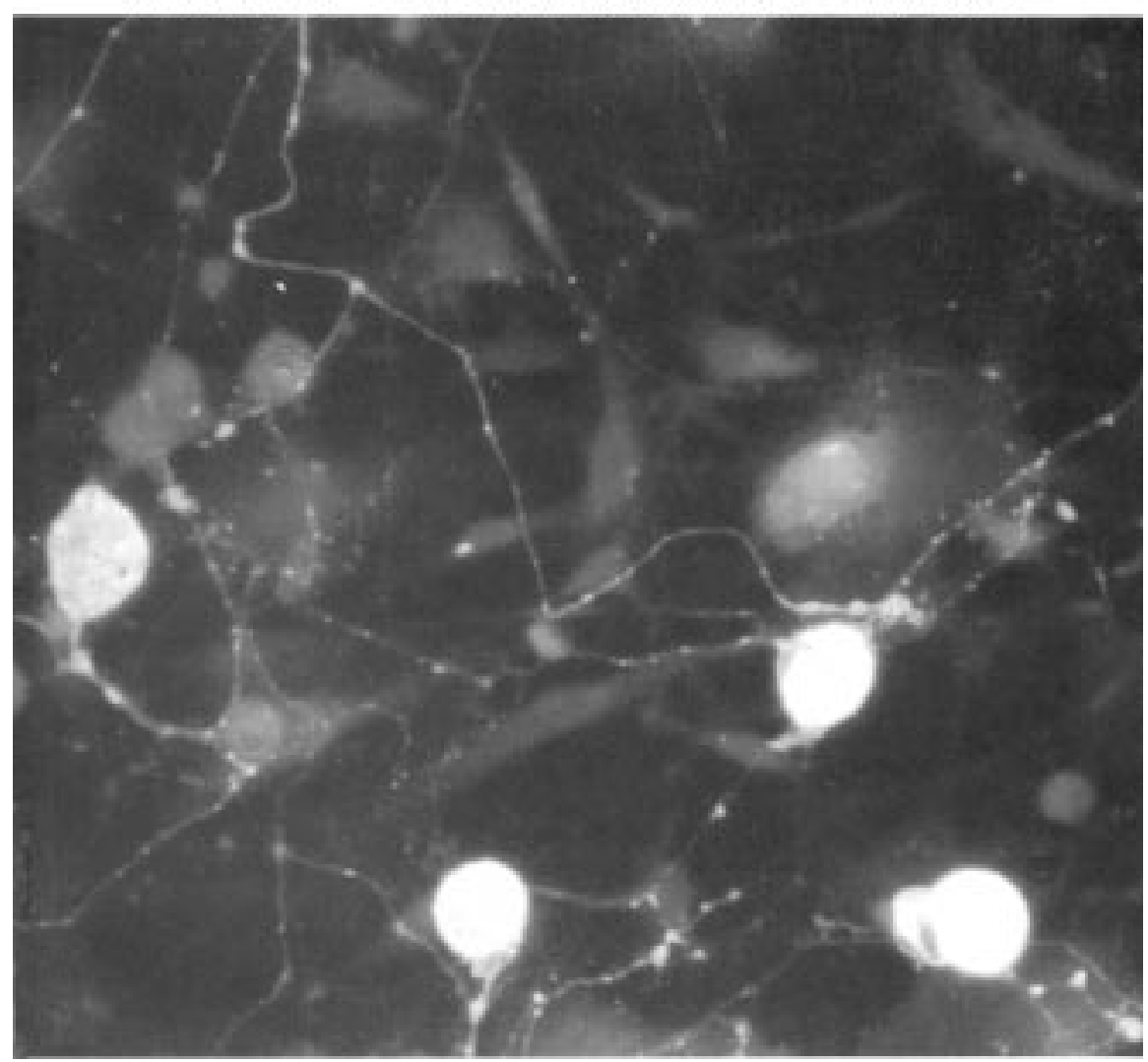

